\title{
12/15-LOX inhibition ameliorates hippocampus associated neuronal damage and mitochondrial dysfunction in mice subjected to hypobaric hypoxia
}

A. Katyal ${ }^{1}$, R. Choudhary ${ }^{2}$

\section{ABSTRACT}

Background and Aim :Oxidative stress is thought to be the critical effector in hypobaric hypoxia induced cognitive dysfunctions. 12/15 Lipoxygenase (12/15 LOX) has recently been described as potent mediator of oxidative stress and is closely associated with cognitive decline. The present study was designed to decipher the underlying role of $12 / 15$ LOX in hypobaric hypoxia induced memory impairment and neuronal damage.

Method: Balb/c mice were subjected to hypobaric hypoxia, simulating condition at $7620 \mathrm{~m}$ altitude. Baicalein(12/15 LOX Inhibitor)was administered to mice. Behavioral paradigm, histopathological assessment and mitochondrial integrity were assessed to establish the involvement of $12 / 15$ LOX in the hypobaric hypoxia neuropathology.

Results:Hypobaric hypoxia episode was accompanied by an increased level of 12/15 LOX and its metabolite 12(S) HETE. The hippocampus CA3 region was found to be mostly affected and showed sign of cellular apoptosis as characterized by elevated activity of caspase-3, 9 \& 8. Working memory impairment seen in mice after hypobaric hypoxia was attenuated following baicalein treatment along with reduced level of caspase activation and HIF-1a. Further, impediment of 12/15 LOX decreased NO level by down-regulating the expression of iNOS, nNOS but not eNOS. A significantly elevated level of cytochrome C was associated with increased 12/15 LOX colocalisation with mitochondria that got reversed following 12/15 LOX inhibition.

Conclusion:12/15 LOX influences the hypobaric hypoxia pathology and its inhibition using baiclein was found to be neuroprotective.

\section{INTRODUCTION}

Decrease in partial pressure of $\mathrm{O}_{2}$ /hypobaric hypoxia results in decline in memory functions associated with increased oxidative stress and neuronal apoptosis in hippocampus. Recently, 12/15LOX emerged as an important amplifier of oxidative stress and has been found to be crucially associated with neurodegenerative conditions including stroke ${ }^{1 .}$. The present study explores the mechanistic insights into the involvement of 12/15-LOX in hypobaric hypoxia induced cognitive impairment and neuronal damage.

\section{OBJECTIVES}

* To evaluate the involvement of $12 / 15$ LOX in hypobaric hypoxia induced working memory deficits and neuronal damage.

* To estimate the relative expression and activity of $12 / 15-$ LOX in hippocampus during hypobaric hypoxia and its modulation by baicalein. - To elucidate the role of 12/15-LOX in modulation of hippocampal hypoxia inducible factor-1a (HIF-1 $\alpha$ ) expression and its targeted genes viz. NOS isoforms during hypobaric hypoxia.

* To investigate the association of 12/15-LOX in executing mitochondria dependent cell death cascade during hypobaric hypoxia.

\section{EXPERIMENTAL PLAN}

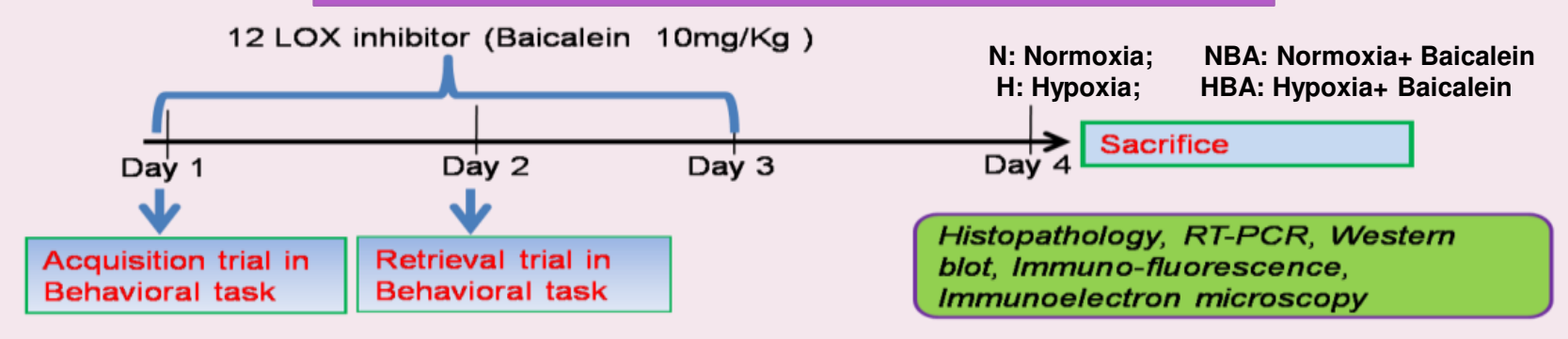

\section{RESULTS}

Baicalein ameliorates hypobaric hypoxia induced memory dysfunction/ oxidative stress and prevents Cytochrome $\mathrm{C}$ release in cytosol
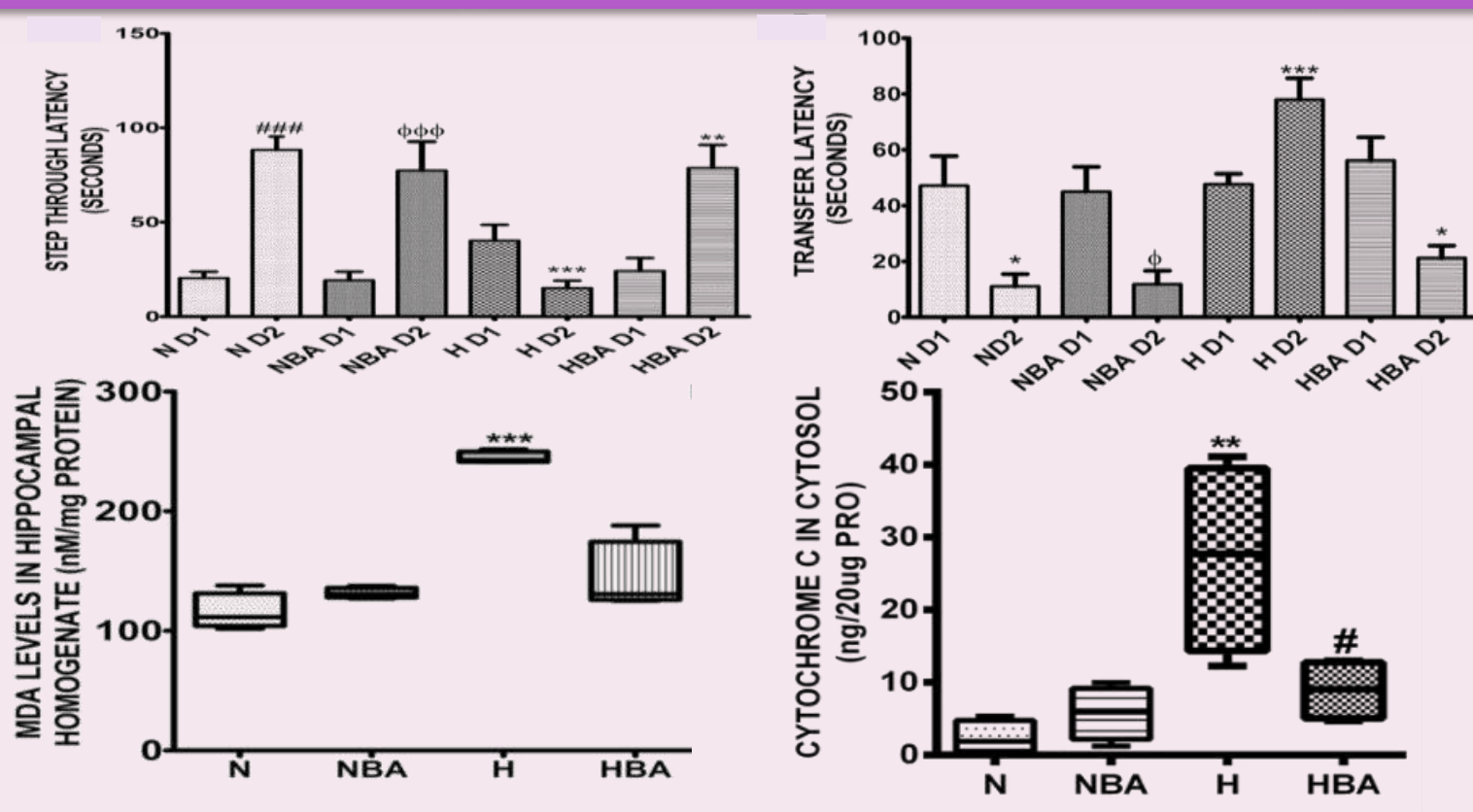

Baicalein reverses alteration in 12/15-LOX and HETE in hypobaric hypoxia

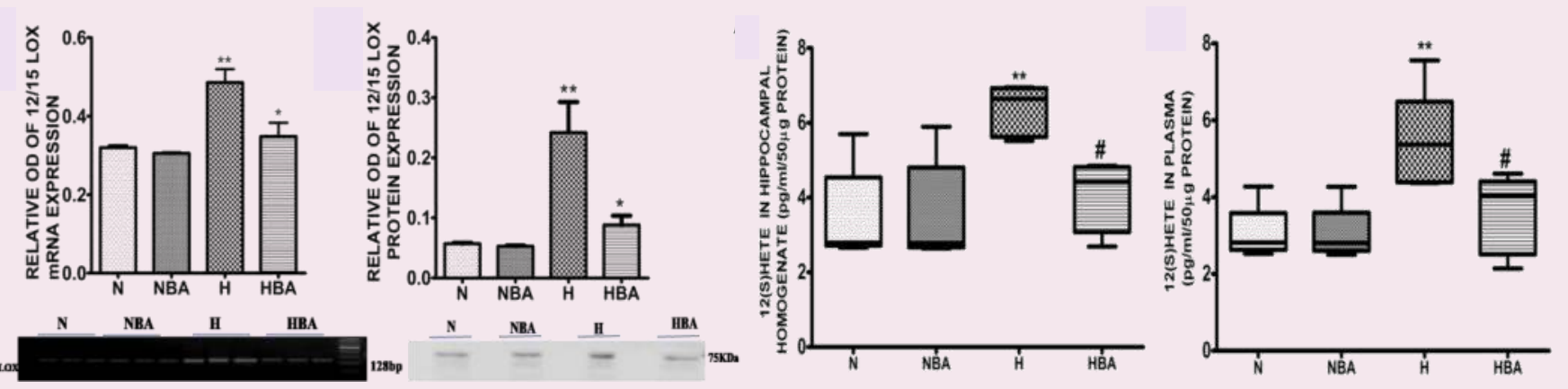

Baicalein mitigates hypobaric hypoxia induced neurotoxicity

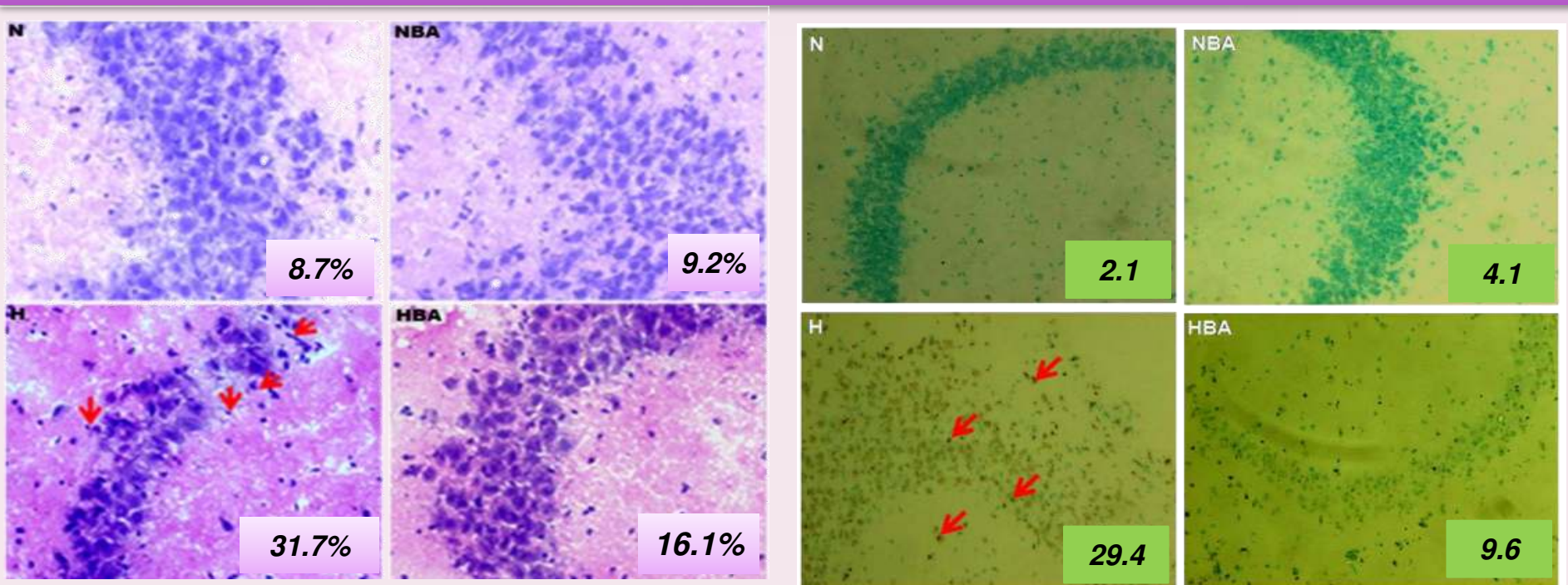

Role of 12/15-LOX in modulation of HIF-1 $\alpha$ and targeted genes (NOS)
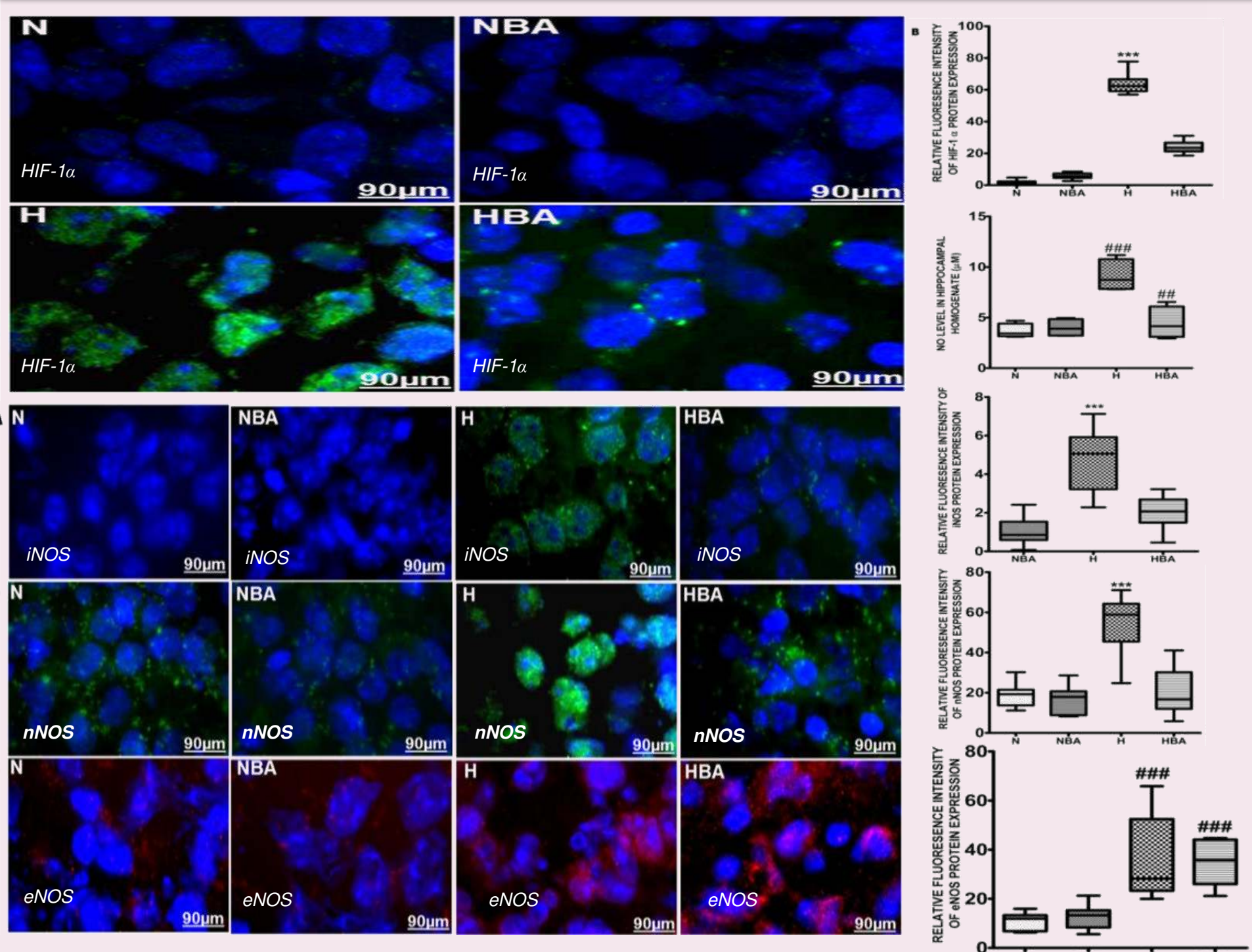

12/15-LOX attacks on the periphery of mitochondria promoting apoptosis
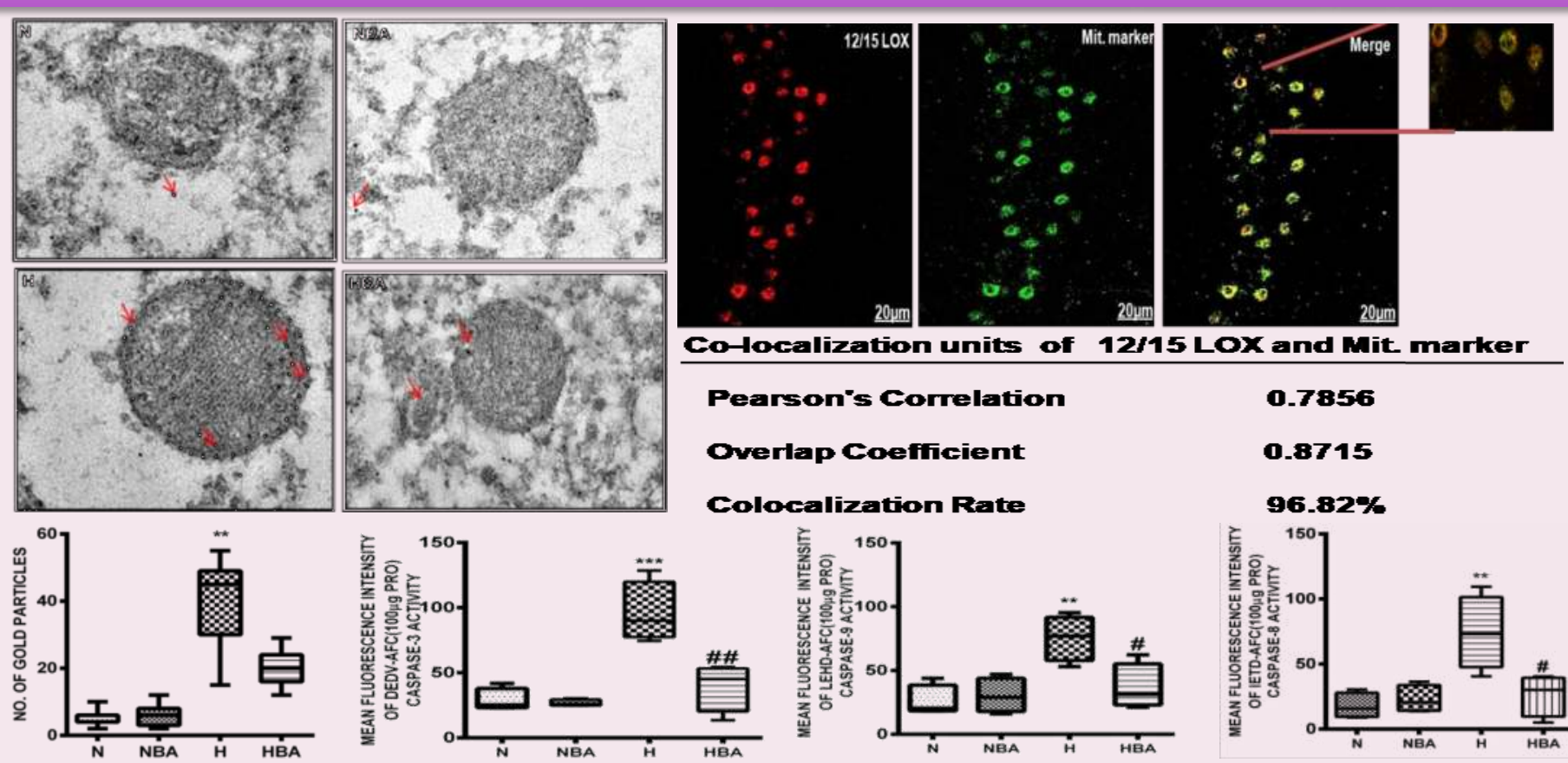

$06.82 \%$

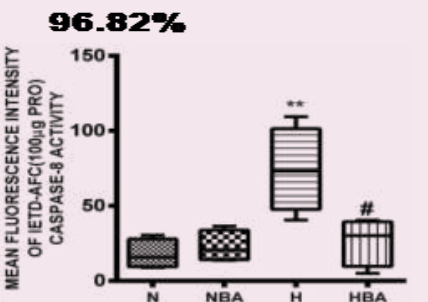

\section{CONCLUSION}

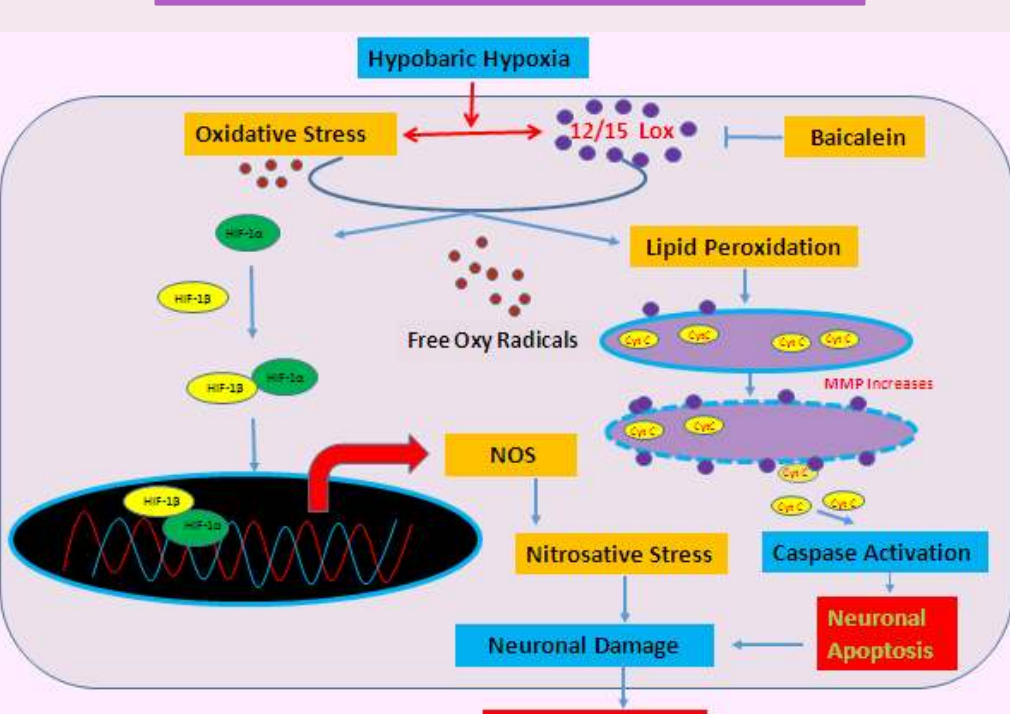

\section{REFERENCES}

1) van Leyen $K$, Kim HY, Lee $\mathrm{SR}$, Jin G, Arai K, Lo EH. Stroke. 37(2006):3014-8.

2) Praticò $D$ etal., Am J

Pathol.164(2004):1655-62.

ACKNOWLEDGEMENT

Authors acknowledge ICMR and CSIR, India for financial support. 